\title{
Modified HScore Predicts Increased Length of Stay but not Mortality in ICU Patients with COVID-19- A Retrospective Cohort Study
}

\author{
Jaskirat $K$ Gill, MD', Megan G Anders, MD, MS', Adnan T Bhutta, MD³, Miranda Gibbons, BS ${ }^{4}$, Peter \\ Rock, MD MBA ${ }^{2}$, Thomas Scalea, $M D^{1}$ and Ali Tabatabai, $M D^{5}$
}

${ }^{1}$ Department of Surgery, Program in Trauma, University of Maryland Medical Center, USA

${ }^{2}$ Department of Anesthesiology, University of Maryland School of Medicine, USA

${ }^{3}$ Department of Pediatrics, University of Maryland School of Medicine, USA

${ }^{4}$ Clinical Submission Analyst, University of Maryland School of Medicine, USA

${ }^{5}$ Division of Pulmonary and Critical Care Medicine, Department of Medicine, University of Maryland School of Medicine, USA

*Corresponding author: Jaskirat Gill, MD, Department of Surgery, Program in Trauma, University of Maryland Medical Center, Baltimore, Maryland, T1R51, 22 S Greene St, Baltimore, MD 21201, USA

\begin{abstract}
Hemophagocytic Lymphohistiocytosis (HLH) is an acute and rapidly progressive systemic inflammatory disorder that can be classified into primary $\mathrm{HLH}(\mathrm{pHLH})$, which is a pediatric disease, and secondary $\mathrm{HLH}(\mathrm{sHLH})$, which can be associated with infection, malignancy, systemic diseases or drugs [1]. The disease is characterized by cytopenia, excessive cytokine production, and hyperferritinemia, and clinically presents with ongoing fevers, lymphadenopathy, hepatosplenomegaly, and multiorgan failure. Of these characteristics, impaired cytotoxic cell function is perhaps the most important and leads to massive cytokine release and a cytokine storm [2]. In 2014 Fardet, et al. developed the HScore which combines physical exam findings and laboratory values to aid in the diagnosis of $\mathrm{HLH}$ with a $93 \%$ sensitivity and $86 \%$ specificity $[2,3]$.

Viral infections are the predominant cause of $\mathrm{sHLH}$, with the most recent inclusion of severe acute respiratory syndrome from coronavirus 2 (SARS-CoV-2), or COVID-19. Overall hospital mortality from COVID-19 early on in the disease was reported to be approximately $15-20 \%$, with up to $40 \%$ of patients requiring ICU admission whereas more recent mortality numbers range from $10-20 \%[4,5]$. The wider range can be attributed to proportionally more younger patients being affected later in the course, additional experience over time and the widespread use of therapies such as prone positioning, steroids and immune modulators.
\end{abstract}

While an association between elevated HScore and adverse outcomes in COVID-19 patients has been proposed, its utility as a risk-stratification tool has not been established in patients with COVID-19 [6]. We therefore undertook this study to investigate whether HScore within the first 24 hours of admission can be used as a prognostic tool to predict outcomes of critically ill adults with COVID-19. We hypothesized that a higher score may be associated with higher mortality.

\section{Keywords}

Hemophagocytic Lymphohistiocytosis, HScore, COVID-19, CRRT

\section{Introduction}

Severe COVID-19 disease is associated with a rapidly progressive systemic inflammatory response similar to that seen in secondary Hemophagocytic Lymphohistiocytosis (sHLH). The HScore is a scoring system used to help diagnose the presence of $\mathrm{sHLH}$. We investigated whether modified HScore $(\mathrm{mHS})$ can be used within the first 24 hours of admission to the ICU to predict morbidity and mortality in critically ill adults with COVID-19. 


\section{Methods}

\section{Patient selection}

We conducted a single-center, retrospective review of medical records of all adult patients (18 and older) admitted to Intensive Care Units at the University of Maryland Medical Center for treatment of COVID-19 on or before May 13, 2020. Patients were either admitted directly from the Emergency Room or were transferred from other hospitals. We only included patients in whom outcome data was available. Patients not admitted to an ICU during their admission or who did not have complete set of laboratory data were excluded. The study was approved by the Institutional Review Board at the University of Maryland, Baltimore.

\section{Data collection}

We collected demographic data (age, gender, race, BMI) as well as clinical data (lab values, admission diagnosis, history of diabetes mellitus and hypertension, known history of HIV, use of ACE inhibitors, ARB agents, or immunosuppressive medications). We also collected data on length of ICU and hospital stay, disposition at discharge, and use of mechanical ventilation (MV), Continuous Renal Replacement Therapy (CRRT) and Extracorporeal Membrane Oxygenation (ECMO). An IRB-approved database was used to access clinical data.

\section{Modified HScore}

HScore is a validated tool for diagnosis of $\mathrm{HLH}$ and its use has been suggested in COVID-19 patients due to the overlap of many clinical and laboratory features (Table 1) $[3,4]$. None of our patients had bone marrow aspiration performed, and presence or absence of organomegaly was infrequently documented. Therefore, we chose to exclude these features and use a modified HScore (mHS) without the points attributed to evidence of hemophagocytosis on bone marrow examination or the points attributed to presence of splenomegaly or hepatomegaly within the first 24 hours of admission.

The $\mathrm{mHS}$ was calculated using a freely available online calculator at http://saintantoine.aphp.fr/score/
[7]

\section{Results}

A total of 125 patients fulfilled the criteria for inclusion in the analysis. The demographic and baseline characteristics of patients are detailed in Table 1. Eightynine out of the 125 patients survived to discharge. The median hospital length of stay was 25.3 days (IQR 12-44 days).

\section{Modified HScore and Mortality}

There was no significant difference between the modified HScore at admission in patients who survived and expired (88.9 vs. 88.9 , p-value 0.988 ) and a ROC curve for mortality with the modified HScore at admission as the only variable showed an area under the curve of 0.48 . The only predictor for mortality on univariate analysis was age at admission (median age in years [IQR]- expired 56 [44.5-64.5] vs survived 48[3458]; OR $\{95 \% \mathrm{Cl}\}, p$-value $=1.04\{1.01-1.06\}, 0.011) . \mathrm{A}$ multivariate logistic regression after stepwise selection showed that in addition to older age as a predictor of mortality [OR 95\% Cl), p-value -1.05 (1.02-1.08), 0.001)], the presence of a pre-existing diagnosis of hypertension conferred a significant survival benefit [No hypertension vs hypertension OR $(95 \% \mathrm{Cl})$, p-value - 1 vs. 0.19 (0.060.61), 0.005].

\section{Modified HScore and Morbidity}

Length of stay: A multiple linear regression with stepwise selection showed that age at admission, hypertension and HScore were significant predictors of the log of length of stay. A significant regression equation was found $(F 93,119)=3.39, p$-value $=0.02)$ with an $\mathrm{R}$ square of 0.08 . Patient's predicted $\log (\mathrm{LOS})$ is equal to $2.6+0.01$ (Age at admission) -0.47 (HTN) +0.006 (H-Score), where hypertension was coded as either 0 (no hypertension or HTN) or 1 (with HTN). Patient's LOS increased $0.6 \%$ for each unit of $\mathrm{H}$-score, each one-year increase in age increased LOS by $2 \%$, and the LOS of patients with HTN was $40 \%$ lower compared to patients without HTN.

Table 1: Scoring system for HScore with associated points.

\begin{tabular}{|l|l|}
\hline Parameter & No.of points (criteria for scoring) \\
\hline Known underlying immunosuppresion & $0(\mathrm{no})$ or 18 (Yes) \\
\hline Temperature $\left({ }^{\circ} \mathrm{C}\right)$ & $0(<38.4), 33(38.4-39.4)$, or $49(>39.4)$ \\
\hline Organomegaly & $\begin{array}{l}0(\mathrm{no}), 23 \text { (hepatomegaly or splenomegaly), or } 38 \text { (hepatomegaly or } \\
\text { splenomegaly) }\end{array}$ \\
\hline No of cytopenias & $0(1$ lineage), $24(2$ lineage), or 34 (3 lineage) \\
\hline Ferritin $(\mathrm{ng} / \mathrm{ml})$ & $0(<2,000), 35(2,000-6,000)$, or $50(>6,000)$ \\
\hline Triglyceride $(\mathrm{mmoles} /$ liter) & $0(<1.5), 44(1.5-4)$, or $64(>4)$ \\
\hline Fibrinogen $(\mathrm{gm} /$ liter) & $0(>2.5)$ or $30(\leq 2.5)$ \\
\hline Serum glutamic oxalocetic transaminase (lu/liter) & $0(<30)$ or $19(\geq 30)$ \\
\hline Hemophagocytosis features on bone marrow aspirate & $0(\mathrm{no})$ or 35 (yes) \\
\hline
\end{tabular}


Length of mechanical ventilation: The $\mathrm{H}$-score did not predict length of mechanical ventilation. The only predictor for length of mechanical ventilation on univariate logistic regression in our cohort was age at admission (OR $=0.95,95 \% \mathrm{Cl} 0.91-0.99$, p-value -0.018). A multivariate logistic regression with stepwise selection showed that increase in each year in age led to a change in ventilator use by a factor of 0.95 .

Use of Continuous Renal Replacement Therapy (CRRT) and Extracorporeal Life Support (ECLS): HScores were significantly different for patients who used CRRT vs those who did not. A multivariate logistic regression with stepwise selection showed that only HScore was associated with CRRT use with an OR of $1.01((5 \% \mathrm{Cl}$, 1.00-1.02, $p$-value $=0.025)$. For each unit increase of HScore, the odds of CRRT use increased by $1 \%$. HScores did not predict utilization of other types of ECLS. The only significant predictors of ECLS use were age at admission and gender. The odds of use of ECMO in men were 3 times that of the odds in women. For increase in each year of age, the odds of ECMO use went down by a factor of 0.96 , holding all other variables constant.

Association between component laboratory values of $\mathbf{H}$ scores and mortality: Of the various component laboratory tests incorporated into the HScore, only the minimum value of the total white blood cell count was significantly different between patients who survived vs those who died (Median [IQR] 9[6.9-142] vs. 12.6\{9.617.2], $\mathrm{p}$ value $=0.008$ ). No significant difference was noted on a multivariate logistic regression.

\section{Discussion}

In this single center retrospective cohort study including 125 patients we found that using the modified HScore does not predict mortality in critically ill adults with COVID-19. There was however an association between higher scores and longer lengths of stay, as well as the need for therapies like CRRT. Approximately $30 \%$ of the cohort did not survive to discharge, which is similar to current ICU mortality with COVID-19 [5]. Age was associated with higher mortality but interestingly a history of hypertension offered a survival benefit. HScores reliably predicted need for CRRT but no other ICU level therapies such as ventilator support or other ECLS modalities such as ECMO. Finally, LOS could also be predicted by HScore.

The association between COVID-19 and HLH continues to be a highly debated topic, with proponents arguing that it is a form of $\mathrm{sHLH}$, while others arguing the similarities only exist due to systemic inflammation. The timeline and severity of disease can impact the expression of pro-inflammatory markers and cytopenias which further complicates the classification. More generally, patients with severe COVID-19 may have a cytokine storm syndrome, the early detection and treatment of which has significant morbidity and mortality implications [8]. In this context, HScore has been suggested as a diagnostic modality [6]. While the immune system plays a key role in virus elimination, its over activation also contributes to the development of ARDS and systemic inflammation. Inability to clear antigenic stimulation leads to inappropriate immune upregulation and a self-sustaining hyper-inflammatory condition known as the cytokine storm, characterized by excessive and persistently high levels of IL-1, IL-2, IL-6, IL-12, IL-16, IL-18, TNF- $\alpha$, and IFN- $\gamma$. The patient's prognosis worsens as the levels of these cytokines increase [2].

The persistent elevation of cytokines leads to increased phagocytosis of germ line cells in the bone marrow and various cytopenias. Both of these are present in the calculation of the HScore, and can be used to argue for immunosuppressive therapy. As none of our patients had bone marrow aspirations and organomegaly was poorly reported we excluded them from our analysis and used the modified HScore (mHS) instead. The mean mHS in our study was 88.9 (SD 35.5), whereas an HScore of 169 is $93 \%$ sensitive and $86 \%$ specific for $\mathrm{HLH}$ [6]. Even if we assume our patients did have organomegaly (38 points) and phagocytosis on bone marrow aspiration (35 points) it would still not reach 169 points. Furthermore, HScore accounts for cytopenias but lacks granularity within cytological lines. Severe COVID-19 is associated with leukocytosis with subset lymphopenia, which is not reflected in the HScore. These findings would therefore argue against HScore being sensitive in COVID-19 [9] and therefore a modified score was used.

As seen in Table 1, our cohort shared many features found in sHLH and cytokine storm syndromes (male preponderance, Age > 50, existing hypertension and diabetes mellitus). We found that mortality was significantly associated with increasing age and, unexpectedly, the absence of pre-existing diagnosis of hypertension but not with mHS. These demographic and clinical features are similar to other studies that have assessed cytokine release syndrome and $\mathrm{HLH}$, and speaks further to the heterogenous features of COVID-19 $[1,10]$. Age, history of hypertension and $\mathrm{mHS}$ also served to predict length of stay in the ICU. Interestingly a history of hypertension offered a survival advantage in our cohort. Typically, hypertension was seen in a large majority of hospitalized COVID-19 patients and was attributed to the population being older and having more co-morbidities [11]. Use of ACEi and ARBs was also initially linked to worse outcomes as the spike protein of the SARS-CoV-2 virus attaches to ACE2 receptors to gain entry into cells [4]. It is possible then those patients in our cohort had fewer ACE2 receptors and had a lower disease burden as a result, or that their ACEi medications were simply discontinued at admission as they routinely are. HScore was the only predictor of the need for Continuous Renal Replacement therapy 
Table 2: Demographic and clinical features of all patients with means.

\begin{tabular}{|l|l|}
\hline Variables & Overall (N = 129) \\
\hline Age at admission N Mean (Std) & 129 \\
\hline $\begin{array}{l}\text { Gender } \\
\text { Male (N/\%) }\end{array}$ & $48.5(16.4)$ \\
\hline $\begin{array}{l}\text { Comorbidities } \\
\text { HTN (N/\%) }\end{array}$ & $90(69.8)$ \\
\hline DM (N/\%) & $33(25.6)$ \\
\hline $\begin{array}{l}\text { BMI } \\
\text { N }\end{array}$ & $38(29.5)$ \\
\hline Mean (Std) & 129 \\
\hline Long-term use of immunosuppressants (N/\%) & $33.3(8.4)$ \\
\hline Long-term use of ACE inhibitors or ARB agent (N/\%) & $13(10.1)$ \\
\hline $\begin{array}{l}\text { H-Score } \\
\text { N }\end{array}$ & $23(17.8)$ \\
\hline Mean (Std) & 129 \\
\hline $\begin{array}{l}\text { Hospital Length of Stay (LOS) } \\
\text { N } \\
\text { Mean (Std) }\end{array}$ & $88.9(35.5)$ \\
\hline
\end{tabular}

Table 3: Comparison of variables based on mortality status.

\begin{tabular}{|l|l|l|l|l|}
\hline Variables & $\begin{array}{l}\text { Overall } \\
(\mathbf{N}=\mathbf{1 2 9})\end{array}$ & $\begin{array}{l}\text { Expired } \\
\mathbf{( N = 3 6 )}\end{array}$ & $\begin{array}{l}\text { Survived } \\
\mathbf{( N = 9 3 )}\end{array}$ & P-value \\
\hline $\begin{array}{l}\text { Modified HScore_at Admission } \\
\text { N }\end{array}$ & 129 & 36 & 93 & 0.998 \\
Mean(std) & $88.9(35.5)$ & $88.9(38.9)$ & $88.9(34.3)$ & \\
\hline
\end{tabular}

speaking to the systemic effects of a hyperinflammatory state. Worsening renal dysfunction would have further led to our patients not being on their home ACEi.

Our primary outcome was survival to discharge, and we found no difference in $\mathrm{mHS}$ between patients who survived vs expired (Table 2). LOS was significantly associated with $\mathrm{mHS}$, where each unit of HScore increased LOS by $0.6 \%$ (Table 3 ). Also, for each unit increase in HScore there was a $1 \%$ increase in CRRT usage. Managing CRRT machines is a labor intense task requiring skilled one to one nursing care. In a COVID positive patient this can impact everything from PPE usage, risk of exposure to staff, and sanitation of machines. Ultimately, it may also account for the increased LOS.

The association between COVID-19 and cytokine release syndromes provides further credence to using immune modulating therapy. The WHO recently published the results from a prospective meta-analysis assessing the efficacy of IL-6 antagonists in patients hospitalized for COVID-19 and found that their usage was associated with a lower 28-day mortality [12-14]. Most of the trials that were included used tocilizumab $(19 / 27)$ and the cohort was again found to have a male predominance with median age being 61. Importantly, IL-6 usage was associated with fewer patients progressing to kidney replacement therapy, which in conjunction with our results advocates for its usage in severe COVID-19 infections, and especially in those with progressive disease [8,15-18].

\section{Limitations}

Our study is limited by being a single center, retrospective analysis of two disease processes that share many features, at time making the distinction difficult. Furthermore, we had to use a modified version of the validated HScore scoring system as our patients did not have bone marrow aspirates or documented organomegaly. While larger trials on steroids, ACEi and immune modulator therapy usage in COVID-19 have shown mortality benefits, our cohort is comparatively smaller and could have factored into not being able to predict mortality.

\section{Conclusion}

COVID-19 has many physical exam and clinical features that are common to $\mathrm{SHLH}$, and its early detection has important implications for initiation of immunosuppressive medications. Modified HScore, a scoring system used to diagnose $\mathrm{sHLH}$, does not predict mortality in patients with COVID-19, but higher scores are associated with increased hospital length of stay. Higher modified HScore is the only predictor of the 


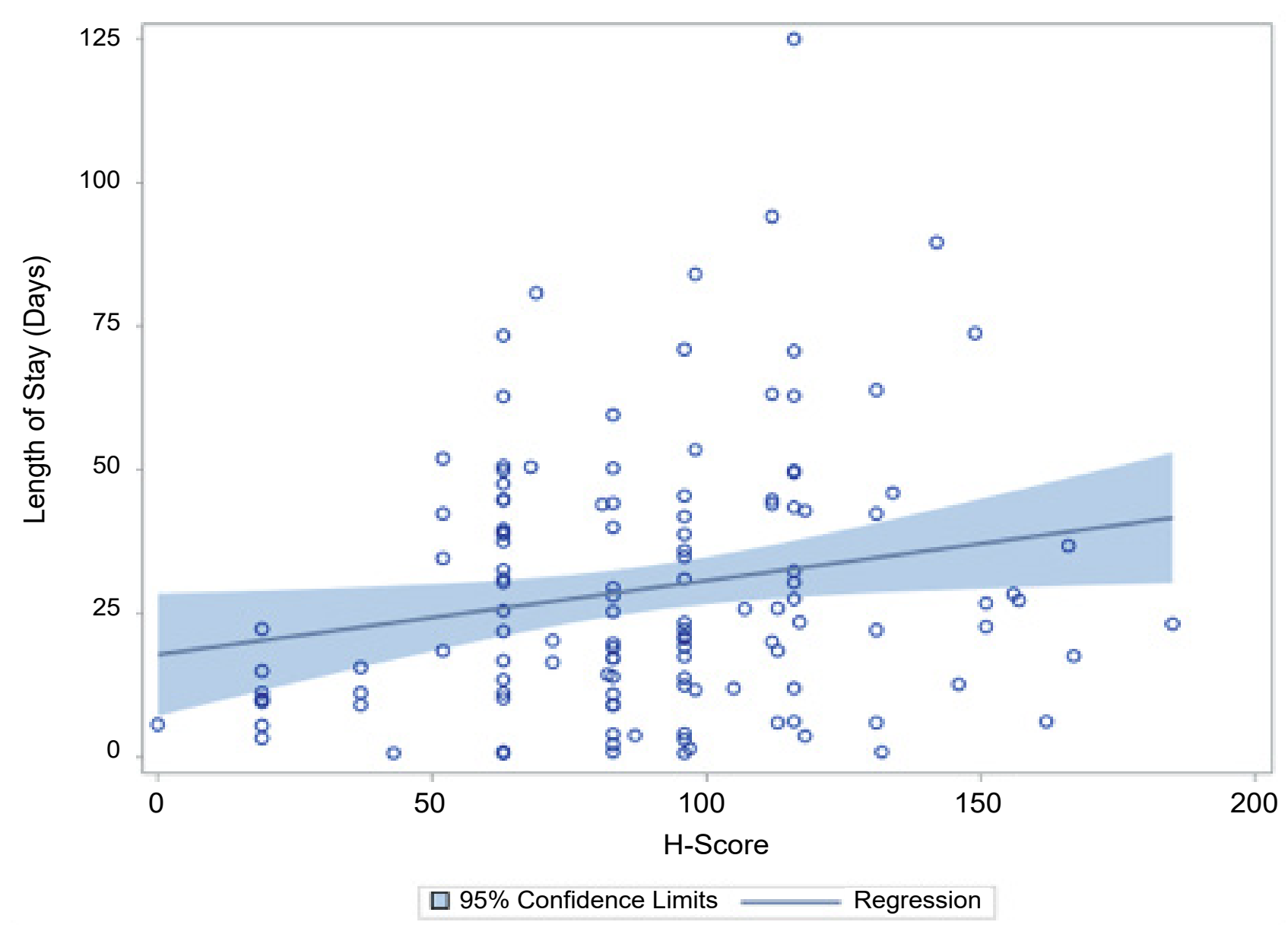

Figure 1: The result of multiple linear regression with backward stepwise selection method indicates $\mathrm{H}$-score is the only predictor significantly associated with LO.

Table 4: Correlation of LOS and continuous variables.

\begin{tabular}{|l|l|}
\hline Variables & LOS \\
\hline & $\begin{array}{l}\text { Pearson Correlation Coefficients } \\
\mathbf{( 9 5 \% ~ C I ) , ~ P - v a l u e ~}\end{array}$ \\
\hline Age at admission & $-0.09(-0.26-0.08), 0.301$ \\
\hline BMI & $-0.13(-0.30-0.04), 0.144$ \\
\hline H-Score & $0.20(0.03-0.36), 0.023$ \\
\hline
\end{tabular}

need for CRRT in our patient population, speaking to its systemic inflammatory effects.

\section{References}

1. Retamozo S, Brito-Zerón $P$, Sisó-Almirall A, Flores-Chávez A, Soto-Cárdenas MJ, et al. (2021) Haemophagocytic syndrome and COVID-19. Clin Rheumatol 40: 1233-1244.

2. Soy M, Atagündüz $P$, Atagündüz I, Sucak GT (2020) Hemophagocytic lymphohistiocytosis: A review inspired by the COVID-19 pandemic. Rheumatol Int 25: 1-12.

3. Fardet L, Galicier L, Lambotte O, Marzac C, Aumont C, et al. (2014) Development and validation of the HScore, a score for the diagnosis of reactive hemophagocytic syndrome. Arthritis Rheumatol 66: 2613-2620.

4. Wiersinga WJ, Rhodes A, Cheng AC, Peacock SJ, Prescott HC (2020) Pathophysiology, Transmission, Diagnosis, and Treatment of Coronavirus Disease 2019 (COVID-19): A Review. JAMA 324: 782-793.

5. Finelli L, Gupta V, Petigara T, Yu K, Bauer KA, et al. (2021)
Mortality Among US Patients Hospitalized With SARSCoV-2 Infection in 2020. JAMA Netw Open 4: e216556.

6. Mehta P, McAuley DF, Brown M, Sanchez E, Tattersall RS, et al. (2020) COVID-19: Consider cytokine storm syndromes and immunosuppression. Lancet 395: 1033-1034.

7. http://saintantoine.aphp.fr/score/

8. Matthay MA, Luetkemeyer AF (2021) IL-6 Receptor Antagonist Therapy for Patients Hospitalized for COVID-19: Who, When, and How?. JAMA 326: 483-485.

9. Lorenz G, Moog P, Bachmann Q, La Rosée P, Schneider H, et al. (2020) Title: Cytokine release syndrome is not usually caused by secondary hemophagocytic lymphohistiocytosis in a cohort of 19 critically ill COVID-19 patients. Sci Rep 10: 18277 .

10. Carvelli J, Demaria O, Vély F, Batista L, Cordier PY, et al. (2020) Association of COVID-19 inflammation with activation of the C5a-C5aR1 axis. Nature 588: 146-150.

11. Leverenz DL, Tarrant TK (2020) Is the HScore useful in COVID-19? Lancet 395: e83.

12. Richardson S, Hirsch JS, Narasimhan M, et al. (2020) Presenting characteristics, comorbidities, and outcomes among 5700 patients hospitalized with COVID-19 in the New York City Area. JAMA 323: 2052-2059.

13. Loscocco GG (2020) Secondary hemophagocytic lymphohistiocytosis, HScore and COVID-19. Int J Hematol 112: $125-126$.

14. Loscocco GG, Malandrino D, Barchiesi S, Berni A, Poggesi L, et al. (2020) The HScore for secondary hemophagocytic 
lymphohistiocytosis, calculated without a marrow biopsy, is consistently low in patients with COVID-19. Int J Lab Hematol 42: e270-e273.

15. WHO Rapid Evidence Appraisal for COVID-19 Therapies (REACT) Working Group, Manu Shankar-Hari, Claire L Vale, Peter J Godolphin, David Fisher, et al. (2021) Association between administration of IL-6 antagonists and mortality among patients hospitalized for COVID-19: A meta-analysis. JAMA 326: 499-518.

16. Yongzhi X (2021) COVID-19-associated cytokine storm syndrome and diagnostic principles: An old and new Issue. Emerg Microbes Infect 10: 266-276.
17. RECOVERY Collaborative Group, Horby P, Lim WS, Emberson JR, Mafham M, et al. (2021) Dexamethasone in Hospitalized Patients with Covid-19. N Engl J Med 384: 693-704.

18. Villar J, Ferrando C, Martínez D, Ambrós A, Muñoz T, et al. (2020) Dexamethasone treatment for the acute respiratory distress syndrome: A multicentre, randomised controlled trial. Lancet Respir Med 8: 267-276. 